\title{
AVALIAÇÃO DOS PROGRAMAS DE PÓS-GRADUAÇÃO EM ENGENHARIA DA UFRJ EMPREGANDO UMA VARIANTE DESENVOLVIDA DO MÉTODO UTA
}

\author{
Luís Alberto Duncan Rangel * \\ UFF-COPPE/PEP/UFRJ \\ duncanuff@hotmail.com
}

Luiz Flávio Autran Monteiro Gomes

Faculdades IBMEC - RJ

autran@ibmecrj.br

Marcos Pereira Estellita Lins

Saul Fuks

COPPE/PEP/UFRJ

lins@pep.ufrj.br

* Corresponding author/autor para quem as correspondências devem ser encaminhadas

Recebido em 05/2002, aceito em 05/2003 após 1 revisão

\begin{abstract}
Resumo
O processo de avaliação em uma organização é geralmente muito complexo, principalmente no meio acadêmico, onde estão presentes diversos interesses, desempenho dos cursos, imagem perante a classe acadêmica e recebimento de recursos, entre outros. Este artigo procura estimar as funções de utilidades dos critérios: pesquisa, horas em sala de aula, orientação e administração, definidos pela Comissão de Avaliação de Docentes da Universidade Federal do Rio de Janeiro, para avaliar os programas de pós-graduação em engenharia da universidade. Neste estudo, empregou-se o método UTA e uma variante desenvolvida do mesmo que considera as diferenças de importância dos critérios empregados na avaliação. Os resultados obtidos com este procedimento mostram funções de utilidades mais próximas das preferências dos decisores, uma vez que essas preferências são incorporadas ao modelo através da inclusão de restrições.
\end{abstract}

Palavras-chave: apoio multicritério à decisão; método UTA; teoria de utilidade.

\begin{abstract}
The evaluation process of human capital in an organization is usually very complex. This is particularly true in the academia, where it is needed to take into account several factors such as the diversity of interests, teaching performance, recognition among his peers and grants received, among others. This paper seeks to estimate the utility functions of the criteria employed in the evaluation process: relevance and quality of research, teaching load, advising activity and administrative tasks, as defined by the "Comissão de Avaliação de Docentes da Universidade Federal do Rio de Janeiro" to evaluate the engineering graduate programs in the University. This study utilizes the UTA method and one of its variants, which considers the different degrees of importance of the criteria employed in the evaluation. This methodology produced utility functions that are very close to the decision makers preferences, since these preferences are incorporated into the model by including the appropriate restrictions.
\end{abstract}

Keywords: multiple criteria decision aid; UTA method; utility theory. 


\section{Introdução}

A Universidade Federal do Rio de Janeiro (UFRJ) possui doze programas de pós-graduação em engenharia, que são avaliados pela Comissão de Avaliação de Docentes (CAD), a qual emprega para este fim, seis critérios.

Este artigo busca determinar as funções de utilidade dos critérios empregados pela CAD, utilizando duas metodologias: o método UTA (Jacquet-Lagrèze \& Siskos, 1982), e uma variante desenvolvida do mesmo método.

O método UTA, de Apoio Multicritério à Decisão (Barba-Romero \& Pomerol, 1997), está baseado em modelos de Programação Linear (PL) especiais, e tem por objetivo determinar: valor global de todas as alternativas presentes na análise, partindo de um conjunto que se refere às alternativas; e as funções de utilidades dos critérios presentes na análise (Vincke, 1989; Zopounidis \& Dimitris, 1998).

Por ser um método da Teoria da Utilidade Multiatributo, todos os critérios são agregados numa única síntese (Roy \& Bouyssou, 1993), e, embora, o UTA original não leve em consideração a diferença de importância dos critérios - uma vez que as constantes de escala podem assumir uma grande diversidade de valores, devido ao conjunto de restrições do modelo matemático - sua variante, aqui utilizada, faz esta consideração, pois cada critério tem efetivamente uma importância para os decisores. Neste artigo, os próprios autores são os decisores.

A variante do método UTA proposta neste estudo, emprega, a priori, a ordenação das alternativas como no método UTA original. A diferença é que nesta variante do método, a ordenação das alternativas a ser utilizada é obtida de modo indireto. Primeiramente, os decisores exprimem suas preferências em relação ao conjunto de critérios, atribuindo-lhe constantes de escala aos mesmos. Empregando o método multicritério de agregação aditiva, determina-se a ordenação das alternativas que, agora, é empregada como informação adicional na implementação do método UTA. Esta variante, também permite incorporar aos modelos, as opiniões dos decisores, em relação ao conjunto de critérios. Com esta finalidade, duas novas restrições são adicionadas às já existentes nos modelos matemáticos, que descrevem o método, uma representando o limite superior e outra o limite inferior, para cada critério, de acordo com as preferências dos decisores, em relação ao conjunto de critérios.

A próxima seção apresenta a variante do método UTA. O estudo de caso é apresentado na seção seguinte onde foram feitas duas análises empregando os dados da CAD; uma utilizando o método UTA original e outra a variante do método UTA. As conclusões deste artigo são apresentadas na última seção. No Apêndice A, apresenta-se o método UTA na formulação original (Jacquet-Lagrèze \& Siskos, 1982).

\section{A Variante do Método UTA}

O conjunto de critérios a serem empregados, no método UTA (Jacquet-Lagrèze \& Siskos, 1982), necessita respeitar os axiomas da Teoria de Utilidade Multiatributo, como por exemplo, a independência em termos da preferência. Existem diversos métodos de Auxílio Multicritério à Decisão de ordenação da alternativa (Gomes, 1998). O método empregado foi o de agregação aditivo dos critérios, apresentado na equação (1), que é a soma da constante de escala de cada critério, multiplicado pelo desempenho das alternativas, em relação a este critério. 


$$
V G\left(a_{i}\right)=\sum_{i}^{n} w_{i} u_{i}\left(a_{i}\right) \quad \text { para todo i e } \mathrm{j}
$$

onde, $V G\left(a_{i}\right)$ é o valor global da alternativa $a_{j}$. Por exemplo, num processo de maximização, quanto maior o valor global de uma alternativa, melhor o seu desempenho, podendo-se ordenar assim as alternativas de forma decrescente, da alternativa de maior valor global para a alternativa de menor valor; $w_{i}$ representa a constante de escala do critério $i$, isto é, a importância desse ponto de vista, aos olhos dos decisores, de acordo com o objetivo do problema abordado; $u_{i}\left(a_{i}\right)$ é o desempenho da alternativa $a_{j}$ em relação ao critério $i$.

Este método de agregação considera a normalização de todos os valores da matriz de avaliação, isto é, o desempenho de cada alternativa, em relação a todos os critérios. Esta normalização é feita para cada critério, dividindo o desempenho de cada alternativa pelo somatório dos desempenhos de todas as alternativas, em relação a este mesmo critério. As constantes de escala dos critérios, empregados neste método de agregação, também, são normalizados.

Para levar em consideração as preferências dos decisores, em relação ao conjunto de critérios nos modelos de programação linear, que descrevem o método UTA, restrições são incorporadas ao mesmo. Duas restrições para cada critério, uma representando o limite superior e outra o limite inferior da constante de escala.

Outro fato importante considerando as preferências dos decisores, é que, é muito mais fácil para os decisores exprimirem suas preferências, em relação a um conjunto pequeno - como os conjuntos de critérios - do que o fazer em relação a grandes conjuntos, como normalmente são os conjuntos das alternativas.

As funções de utilidade estimada sob esta consideração, representam mais fielmente as preferências dos decisores, pois, os modelos matemáticos que descrevem esta variante do método UTA, buscam obter soluções para as variáveis, passíveis de novas restrições que foram incorporadas aos modelos.

Os problemas de programação linear (PPL), PPL1, PPL2 e PPL3 apresentados no apêndice A, descrevem o método UTA original. A estes PPLs incorporam-se as restrições (2) e (3):

$$
\begin{aligned}
& u_{i}\left(g_{i}^{*}\right) \leq \mathrm{w}_{\mathrm{i}}+2 \delta \\
& u_{i}\left(g_{i}^{*}\right) \geq \mathrm{w}_{\mathrm{i}}-2 \delta
\end{aligned}
$$

gerando outros problemas de programação linear PPL1', PPL2' e PPL3'. $\delta$ é um valor pequeno definido pelos decisores, dentro da faixa estipulado pelo método UTA, de acordo com o número de alternativas do problema, a fim de separar duas classes consecutivas das alternativas. Portanto, o PPL1', ao ser implementado sob esta consideração, estará sujeito as restrições (2) e (3), além das restrições do modelo UTA original, apresentados no apêndice A; (18), (19), (20), (21), (22), (23), (24). O PPL2' e PPL3', além destas restrições do PPL1', tem a restrição (25), apresentado no apêndice A.

A inclusão destas restrições busca considerar as preferências dos decisores, aos modelos que descrevem o método UTA, a fim de obter a função de utilidade de cada critério, o mais próximo possível das preferências dos decisores. 


\section{Estudo de Caso}

\subsection{A Comissão de Avaliação de Docentes}

A Comissão de Avaliação de Docentes (CAD) da Coordenação dos Programas de PósGraduação de Engenharia (COPPE) da Universidade Federal do Rio de Janeiro (UFRJ) está encarregada de estabelecer formas de acompanhamento e controle do desempenho do corpo docente e dos grupos de pesquisa da COPPE/UFRJ, sob este propósito, a CAD elaborou um sistema de avaliação anual, que tenta "contemplar múltiplas características desejáveis em docentes de Pós-Graduação compromissados fortemente com a pesquisa científica e tecnológica" (CAD, 1997). Este sistema de avaliação impõe exigências para a progressão na carreira de docente.

No sistema de avaliação da CAD-UFRJ foram estabelecidos instrumentos de pontuação de atividades de ENSINO, ORIENTAÇÃO (de teses), PESQUISA e APOIO, correspondendo estas atividades aos critérios de avaliação de docentes da CAD.

No critério ENSINO, considera-se a carga didática de cada docente em disciplinas de graduação, mestrado e doutorado.

No critério ORIENTAÇÃO de teses, avalia-se o número de teses de mestrado e doutorado orientadas pelo docente e o número de teses efetivamente concluídas.

O critério PESQUISA refere-se à contribuição de pesquisa de cada docente. Este critério é avaliado através de um sistema mais elaborado, baseado, principalmente, em indicadores de qualidade dos veículos de publicações.

O critério APOIO, segundo o sistema de avaliação da CAD-UFRJ, não tem muita influência para a progressão na carreira do docente. Este critério valoriza o esforço realizado pelos docentes em cargos administrativos, em cargos de Direção, Chefia de Departamentos, Organização de Eventos, além de Comissões Internas/Externas. A avaliação deste critério baseia-se no total de horas dedicadas a cada atividade, havendo, no entanto, um limite máximo no número de horas que podem ser consideradas.

Os critérios PESQUISA e ORIENTAÇÃO de teses foram divididos pela CAD-UFRJ em dois sub-critérios, definidos a seguir: a Pesquisa Tipo I, correspondendo a publicações em revistas indexadas pelo I.S.I. (Institute for Scientific Information) e/ou publicações nas revistas mais importantes em cada especialidade, em âmbito internacional; livros com conteúdo original relevante, com distribuição internacional; e, capítulos de livros quando forem equiparáveis tecnicamente às publicações do Tipo I; e a Pesquisa Tipo II refere-se a publicações em revistas não classificadas no Tipo I; publicações em Anais de Conferências; livros de caráter eminentemente didático ou de distribuição nacional; capítulos de livros não classificáveis no Tipo I; e, processos e protótipos com patente.

$\mathrm{O}$ item Teses Totais corresponde a todas as teses orientadas pelo docente, já o critério Teses de Doutorado, só leva em consideração as teses de doutorado orientadas pelo docente.

A CAD-UFRJ, responsável pelo funcionamento do sistema de avaliação, coleta e processa os dados anualmente para a avaliação e, complementa a pontuação com uma avaliação qualitativa de cada docente, tendo em vista um padrão mínimo de desempenho, bem como o perfil mínimo para a promoção. 


\subsection{Implementação}

Os dados empregados neste estudo dizem respeito aos doze programas de pós-graduação em engenharia da UFRJ que foram avaliados pela CAD: Biomédica, Civil, Elétrica, Mecânica, Metalúrgica e de Materiais, Nuclear, Oceânica, Planejamento Energético, Produção, Química, Sistemas e Computação, e Transportes (CAD, 1999). Neste estudo, os decisores são os próprios autores do artigo, pois todos são professores universitários experientes, já tendo trabalhado em diversas avaliações no meio acadêmico.

Os programas são identificados através de letras, e a ordem apresentada na Tabela I não está relacionada com a ordem do parágrafo anterior, para não serem identificados. Os critérios, conforme descrito na introdução, são identificados a seguir: Critério C1 (Pesquisa Tipo I), Critério C2 (Pesquisa Tipo II), Critério C3 (Educação), Critério C4 (Teses Totais), Critério C5 (Teses Doutorado) e Critério C6 (Suporte). Admite-se que o método de agregação empregado pela CAD é desconhecido.

A Tabela I apresenta os impactos médios dos programas obtidos para cada critério nas avaliações feitas em (96/97), (97/98) e (98/99) (CAD, 1999).

Duas implementações foram feitas empregando os dados da CAD. Na primeira implementação, empregou-se o método UTA original (Jacquet-Lagrèze \& Siskos, 1982), considerando como dado de entrada a informação adicional fornecida pelos decisores, que são os pontos obtidos pelos programas e a matriz de avaliação. A condição de independência em termos de preferência necessária ao emprego do método UTA foi garantida através de uma análise feita pelos decisores empregando as teorias de Keeney \& Raiffa (1993).

Tabela I - Matriz dos Programas x Critérios

\begin{tabular}{|c|c|c|c|c|c|c|c|}
\hline \multirow{2}{*}{ Programas } & \multicolumn{7}{|c|}{ Critérios } \\
\cline { 2 - 8 } & C1 & C2 & C3 & C4 & C5 & C6 & Pontos \\
\hline A & 1,14 & 6,75 & 2,57 & 3,29 & 1,37 & 0,97 & 13,58 \\
\hline B & 1,35 & 6,42 & 2,64 & 3,52 & 1,81 & 0,60 & 13,18 \\
\hline C & 2,00 & 5,66 & 2,65 & 2,75 & 0,97 & 0,34 & 11,40 \\
\hline D & 1,11 & 4,45 & 2,04 & 2,84 & 1,25 & 0,58 & 9,92 \\
\hline E & 2,09 & 5,98 & 3,07 & 2,31 & 0,82 & 0,51 & 11,98 \\
\hline F & 2,74 & 6,71 & 2,49 & 3,27 & 1,12 & 0,93 & 13,40 \\
\hline G & 0,39 & 2,63 & 3,04 & 2,64 & 1,06 & 0,74 & 9,05 \\
\hline H & 0,82 & 3,00 & 1,75 & 4,41 & 1,11 & 1,38 & 10,53 \\
\hline I & 0,23 & 2,47 & 2,82 & 5,12 & 2,64 & 0,63 & 11,04 \\
\hline J & 6,45 & 16,36 & 2,41 & 4,06 & 2,21 & 1,45 & 24,28 \\
\hline K & 0,97 & 4,57 & 1,77 & 2,93 & 1,33 & 0,41 & 9,68 \\
\hline L & 0,21 & 5,06 & 1,89 & 3,02 & 0,84 & 0,87 & 10,84 \\
\hline
\end{tabular}

A Tabela II constitui uma mera arrumação dos dados da Tabela I. A Tabela II apresenta os programas ordenados de acordo com seus respectivos desempenhos finais. 
Tabela II - Ordenação dos Programas

\begin{tabular}{|c|c|c|c|c|c|c|}
\hline \multirow{2}{*}{ Programas } & \multicolumn{7}{|c|}{ Critérios } \\
\cline { 2 - 7 } & C1 & C2 & C3 & C4 & C5 & C6 \\
\hline J & 6,45 & 16,36 & 2,41 & 4,06 & 2,21 & 1,45 \\
\hline E & 2,09 & 5,98 & 3,07 & 2,31 & 0,82 & 0,51 \\
\hline C & 2,00 & 5,66 & 2,65 & 2,75 & 0,97 & 0,34 \\
\hline B & 1,35 & 6,42 & 2,64 & 3,52 & 1,81 & 0,60 \\
\hline A & 1,14 & 6,75 & 2,57 & 3,29 & 1,37 & 0,97 \\
\hline D & 1,11 & 4,45 & 2,04 & 2,84 & 1,25 & 0,58 \\
\hline G & 0,39 & 2,63 & 3,04 & 2,64 & 1,06 & 0,74 \\
\hline I & 0,23 & 2,47 & 2,82 & 5,12 & 2,64 & 0,63 \\
\hline L & 0,21 & 5,06 & 1,89 & 3,02 & 0,84 & 0,87 \\
\hline F & 2,74 & 6,71 & 2,49 & 3,27 & 1,12 & 0,93 \\
\hline H & 0,82 & 3,00 & 1,75 & 4,41 & 1,11 & 1,38 \\
\hline K & 0,97 & 4,57 & 1,77 & 2,93 & 1,33 & 0,41 \\
\hline
\end{tabular}

Os valores máximos $\left(\mathrm{g}_{\mathrm{i}}^{*}\right)$ e mínimos $\left(\mathrm{g}_{\mathrm{i}^{*}}\right)$ de cada critério $\mathrm{i}$; o número de pontos $\left(\alpha_{\mathrm{i}}\right)$ considerados na construção de cada função de utilidade correspondente a cada critério i, isto é, os trechos retos que irão representar cada função considerada como sendo linear por partes; bem como a folga $\left(\mathrm{s}_{\mathrm{i}}\right)$ entre pontos consecutivos de cada critério i são apresentados na Tabela III. Os dois últimos parâmetros são definidos pelos decisores.

Outro parâmetro desse método é o $\delta$, empregado nos modelos matemáticos do método UTA, cujo valor no estudo de caso fez-se igual a 0,01. Esse valor foi definido pelos agentes de decisão e pertence ao intervalo sugerido pelos autores do método UTA, (Jacquet-Lagrèze \& Siskos, 1982) [ver Apêndice A] conforme o número de classes Q consideradas no problema abordado. A faixa do valor de $\delta$ sugerida pelos decisores varia de $[0,0083 ; 0,0833]$. Neste estudo, cada alternativa foi considerada como sendo uma classe, como o conjunto de referência possui 12 elementos, portanto, 12 são as classes consideradas neste trabalho. Esta descrição apresentada indica que não existe relação de indiferença entre as alternativas, somente foram consideradas relações de preferência entre as alternativas presentes na análise.

Tabela III - Dados para a implementação do método UTA

\begin{tabular}{|c|c|c|c|c|}
\hline Critérios & $\mathrm{g}_{\mathrm{i}^{*}}$ & $\mathrm{~g}_{\mathrm{i}}{ }^{*}$ & $\alpha_{\mathrm{i}}$ & $\mathrm{s}_{\mathrm{i}}$ \\
\hline $\mathrm{C} 1$ & 0,21 & 6,45 & 5 & 0 \\
\hline $\mathrm{C} 2$ & 2,47 & 16,36 & 5 & 0 \\
\hline $\mathrm{C} 3$ & 1,75 & 3,07 & 5 & 0 \\
\hline $\mathrm{C} 4$ & 2,31 & 5,12 & 5 & 0 \\
\hline $\mathrm{C} 5$ & 0,82 & 2,64 & 5 & 0 \\
\hline $\mathrm{C} 6$ & 0,34 & 1,45 & 5 & 0 \\
\hline
\end{tabular}


A Tabela IV apresenta as constantes de escala dos critérios. A primeira implementação, empregando o método UTA original não considera as constantes escala dos critérios.

Tabela IV - Constante de Escala dos Critérios

\begin{tabular}{|c|c|c|c|c|c|c|}
\hline & \multicolumn{7}{|c|}{ Critérios } \\
\cline { 2 - 7 } & $\mathrm{C} 1$ & $\mathrm{C} 2$ & $\mathrm{C} 3$ & $\mathrm{C} 4$ & $\mathrm{C} 5$ & C6 \\
\hline $\begin{array}{c}\text { Constantes de } \\
\text { Escala }\end{array}$ & 0,00021 & 0,250061 & 0,25017 & 0,25008 & 0,00093 & 0,24830 \\
\hline
\end{tabular}

A função objetivo do método UTA visa minimizar os erros que são associados ao valor global de cada alternativa para obter a ordenação, proposta a priori pelos decisores. As variáveis dos modelos de programação linear do método UTA são aquelas que representam as folgas que são adicionadas ao valor global de cada alternativa e aquelas variáveis que definem a utilidade de cada critério.

Para resolver os problemas de programação linear dos dois métodos analíticos utilizados nesse estudo de caso, o método UTA (Jacquet-Lagrèze \& Siskos, 1982) na sua formulação original e a variante desenvolvida do método UTA, empregou-se o software Lindo.

Os resultados obtidos através do método UTA original, primeira análise, são apresentados na Tabela V. A Coluna - 3 apresenta os resultados das variáveis onde o valor da função objetivo foi igual a zero, implementação do PPL1, indicando que o modelo conseguiu obter a mesma ordenação fornecida pela CAD, não atribuindo nenhum valor diferente de zero as variáveis que são adicionadas ao valor global das alternativas, para auxiliar a ordenação dos programas de acordo com a ordenação da CAD. A Coluna - 4 da Tabela V apresenta os valores médios das variáveis após análise de pós-otimização, implementação dos PPL2 e PPL3.

Tabela V - Comparação dos Resultados

\begin{tabular}{|c|c|c|c|c|c|}
\hline \multirow{3}{*}{ Critério } & \multirow{2}{*}{ Variável } & \multicolumn{2}{|c|}{$\begin{array}{c}\text { Sem considerar as constantes } \\
\text { de escala dos Critérios }\end{array}$} & \multicolumn{2}{c|}{$\begin{array}{c}\text { Considerando as constantes de } \\
\text { escala dos Critérios }\end{array}$} \\
\cline { 3 - 6 } & & $\mathrm{F}=0$ & Pós-Otimização & $\mathrm{F}=0$ & Pós-Otimização \\
\hline Coluna 1 & Coluna 2 & Coluna 3 & Coluna 4 & Coluna 5 & Coluna 6 \\
\hline \multirow{5}{*}{ C1 } & $\mathrm{u}_{1}\left(\mathrm{~g}_{1}{ }^{1}\right)$ & 0,000 & 0,000 & 0,000 & 0,000 \\
\cline { 2 - 6 } & $\mathrm{u}_{1}\left(\mathrm{~g}_{1}{ }^{2}\right)$ & 0,000 & 0,013 & 0,020 & 0,005 \\
\cline { 2 - 6 } & $\mathrm{u}_{1}\left(\mathrm{~g}_{1}{ }^{3}\right)$ & 0,000 & 0,013 & 0,020 & 0,005 \\
\cline { 2 - 6 } & $\mathrm{u}_{1}\left(\mathrm{~g}_{1}{ }^{4}\right)$ & 0,000 & 0,013 & 0,020 & 0,007 \\
\cline { 2 - 6 } & $\mathrm{u}_{1}\left(\mathrm{~g}_{1}{ }^{5}\right)$ & 0,484 & 0,143 & 0,020 & 0,010 \\
\hline \multirow{5}{*}{$\mathrm{C} 2$} & $\mathrm{u}_{2}\left(\mathrm{~g}_{2}{ }^{1}\right)$ & 0,000 & 0,000 & 0,000 & 0,000 \\
\cline { 2 - 6 } & $\mathrm{u}_{2}\left(\mathrm{~g}_{2}{ }^{2}\right)$ & 0,208 & 0,181 & 0,270 & 0,266 \\
\cline { 2 - 6 } & $\mathrm{u}_{2}\left(\mathrm{~g}_{2}{ }^{3}\right)$ & 0,208 & 0,252 & 0,270 & 0,266 \\
\cline { 2 - 6 } & $\mathrm{u}_{2}\left(\mathrm{~g}_{2}{ }^{4}\right)$ & 0,208 & 0,252 & 0,270 & 0,266 \\
\cline { 2 - 6 } & $\mathrm{u}_{2}\left(\mathrm{~g}_{2}{ }^{5}\right)$ & 0,208 & 0,544 & 0,270 & 0,266 \\
\hline
\end{tabular}

(continua...) 
Tabela V (continuação) - Comparação dos Resultados

\begin{tabular}{|c|c|c|c|c|c|}
\hline \multirow[t]{2}{*}{ Critério } & \multirow[t]{2}{*}{ Variável } & \multicolumn{2}{|c|}{$\begin{array}{c}\text { Sem considerar as constantes } \\
\text { de escala dos Critérios }\end{array}$} & \multicolumn{2}{|c|}{$\begin{array}{c}\text { Considerando as constantes de } \\
\text { escala dos Critérios }\end{array}$} \\
\hline & & $\mathrm{F}=0$ & Pós-Otimização & $\mathrm{F}=0$ & Pós-Otimização \\
\hline \multirow{5}{*}{$\mathrm{C} 3$} & $\mathrm{u}_{3}\left(\mathrm{~g}_{3}{ }^{1}\right)$ & 0,000 & 0,000 & 0,000 & 0,000 \\
\hline & $\mathrm{u}_{3}\left(\mathrm{~g}_{3}{ }^{2}\right)$ & 0,081 & 0,029 & 0,119 & 0,103 \\
\hline & $\mathrm{u}_{3}\left(\mathrm{~g}_{3}{ }^{3}\right)$ & 0,081 & 0,032 & 0,130 & 0,126 \\
\hline & $\mathrm{u}_{3}\left(\mathrm{~g}_{3}{ }^{4}\right)$ & 0,081 & 0,039 & 0,146 & 0,126 \\
\hline & $\mathrm{u}_{3}\left(\mathrm{~g}_{3}{ }^{5}\right)$ & 0,081 & 0,057 & 0,230 & 0,231 \\
\hline \multirow{5}{*}{$\mathrm{C} 4$} & $\mathrm{u}_{4}\left(\mathrm{~g}_{4}{ }^{1}\right)$ & 0,000 & 0,000 & 0,000 & 0,000 \\
\hline & $\mathrm{u}_{4}\left(\mathrm{~g}_{4}{ }^{2}\right)$ & 0,000 & 0,034 & 0,160 & 0,158 \\
\hline & $\mathrm{u}_{4}\left(\mathrm{~g}_{4}{ }^{3}\right)$ & 0,068 & 0,040 & 0,160 & 0,158 \\
\hline & $\mathrm{u}_{4}\left(\mathrm{~g}_{4}{ }^{4}\right)$ & 0,131 & 0,082 & 0,160 & 0,158 \\
\hline & $\mathrm{u}_{4}\left(\mathrm{~g}_{4}{ }^{5}\right)$ & 0,131 & 0,125 & 0,236 & 0,253 \\
\hline \multirow{5}{*}{ C5 } & $\mathrm{u}_{5}\left(\mathrm{~g}_{5}^{1}\right)$ & 0,000 & 0,000 & 0,000 & 0,000 \\
\hline & $\mathrm{u}_{5}\left(\mathrm{~g}_{5}^{2}\right)$ & 0,024 & 0,008 & 0,001 & 0,009 \\
\hline & $\mathrm{u}_{5}\left(\mathrm{~g}_{5}^{3}\right)$ & 0,024 & 0,011 & 0,001 & 0,009 \\
\hline & $\mathrm{u}_{5}\left(\mathrm{~g}_{5}{ }^{4}\right)$ & 0,024 & 0,011 & 0,001 & 0,009 \\
\hline & $\mathrm{u}_{5}\left(\mathrm{~g}_{5}^{5}\right)$ & 0,024 & 0,049 & 0,020 & 0,012 \\
\hline \multirow{5}{*}{ C6 } & $\mathrm{u}_{6}\left(\mathrm{~g}_{6}{ }^{1}\right)$ & 0,000 & 0,000 & 0,000 & 0,000 \\
\hline & $\mathrm{u}_{6}\left(\mathrm{~g}_{6}^{2}\right)$ & 0,032 & 0,011 & 0,000 & 0,000 \\
\hline & $\mathrm{u}_{6}\left(\mathrm{~g}_{6}{ }^{3}\right)$ & 0,072 & 0,028 & 0,000 & 0,006 \\
\hline & $\mathrm{u}_{6}\left(\mathrm{~g}_{6}{ }^{4}\right)$ & 0,072 & 0,057 & 0,096 & 0,075 \\
\hline & $\mathrm{u}_{6}\left(\mathrm{~g}_{6}{ }^{5}\right)$ & 0,072 & 0,082 & 0,224 & 0,228 \\
\hline Coluna 1 & Coluna 2 & Coluna 3 & Coluna 4 & Coluna 5 & Coluna 6 \\
\hline
\end{tabular}

A Tabela VI apresenta os valores limites das constantes de escala. A Coluna - 3 apresenta os limites superiores e a Coluna - 4 apresenta os limites inferiores.

Tabela VI - Limites das Constantes de Escala

\begin{tabular}{|c|c|c|c|c|c|}
\hline \multirow{2}{*}{\multicolumn{2}{|c|}{ Critérios }} & \multicolumn{4}{|c|}{ Limites da Constante de Escala de Cada Critério } \\
\cline { 3 - 6 } \multicolumn{2}{|c|}{} & \multicolumn{2}{|c|}{$\begin{array}{c}\text { Sem Considerar as Constantes de } \\
\text { Escala }\end{array}$} & $\begin{array}{c}\text { Considerando as Constantes de } \\
\text { Escala }\end{array}$ \\
\cline { 3 - 6 } & $\mathrm{g}_{1}{ }^{*}$ & 0,00 & 0,81 & 0,00 & 0,02 \\
\hline $\mathrm{C} 1$ & $\mathrm{~g}_{2}{ }^{*}$ & 0,07 & 0,91 & 0,23 & 0,27 \\
\hline $\mathrm{C} 2$ & $\mathrm{~g}_{3}{ }^{*}$ & 0,00 & 0,30 & 0,23 & 0,24 \\
\hline $\mathrm{C} 3$ & $\mathrm{~g}_{4}{ }^{*}$ & 0,00 & 0,46 & 0,23 & 0,27 \\
\hline $\mathrm{C} 4$ & $\mathrm{~g}_{5}{ }^{*}$ & 0,00 & 0,38 & 0,00 & 0,02 \\
\hline $\mathrm{C} 5$ & $\mathrm{~g}_{6}{ }^{*}$ & 0,00 & 0,39 & 0,22 & 0,25 \\
\hline $\mathrm{C} 6$ & Coluna 2 & Coluna 3 & Coluna 4 & Coluna 5 & Coluna 6 \\
\hline Coluna 1
\end{tabular}


A segunda implementação, a variante desenvolvida do método UTA, considerou as constantes de escala dos critérios (Rangel et al., 2000), baseando-se nas informações da avaliação dos programas de pós-graduação em engenharia feita pela $\mathrm{CAD}$, apresentadas na Tabela I. A ordenação dos programas a ser utilizada nesta segunda implementação foi obtida empregando o método de agregação aditivo dos critérios. A ordenação obtida foi a mesma apresentada na Tabela II.

Os limites extremos dos critérios, número de pontos que vão definir a função de utilidade de cada critério e folga entre os pontos consecutivos de um mesmo critério considerados nesta segunda implementação são os mesmos da primeira implementação, apresentados na Tabela III.

O limite superior e inferior considerado para a constante de escala de cada critério, isto é, a faixa de variação do valor extremo máximo de cada critério na segunda implementação, que representa uma folga das constantes de escala, foi adicionado ao modelo buscando restringir o conjunto de soluções ótimas, de modo a obter soluções tão próximas quanto possível às preferências dos decisores. Esses limites foram estabelecidos em função do valor de delta, valor este, definidos pelos agentes de decisão para identificar as classes das alternativas presentes na análise.

Em função desse valor delta estabeleceu-se os valores limites para a constante de escala de cada critério. O limite inferior é obtido diminuindo o valor da constante de escala do critério menos duas vezes o valor de delta, e o limite superior é obtido somando ao valor da constante de escala duas vezes o valor de delta. As restrições (2) e (3) determinam esses valores. A Tabela VII apresenta os limites considerados para cada critério.

Tabela VII - Limites de Variação dos Critérios

\begin{tabular}{|c|c|c|c|c|c|c|}
\hline \multirow{2}{*}{ Limites } & \multicolumn{6}{|c|}{ Critérios } \\
\cline { 2 - 7 } & $\mathrm{C} 1$ & $\mathrm{C} 2$ & $\mathrm{C} 3$ & $\mathrm{C} 4$ & $\mathrm{C} 5$ & C6 \\
\hline Mínimo & 0,00 & 0,23 & 0,23 & 0,23 & 0,00 & 0,22 \\
\hline Máximo & 0,02 & 0,27 & 0,27 & 0,27 & 0,02 & 0,26 \\
\hline
\end{tabular}

Os resultados obtidos através da variante do método UTA, considerando as restrições as constantes de escala, limites superiores e inferiores, são apresentados na Tabela V. A Coluna - 5 desta tabela apresenta os resultados das variáveis obtidos através da implementação do PPL1', onde o valor da função objetivo foi igual a zero, indicando que o modelo conseguiu obter a mesma ordenação fornecida pela CAD. A Coluna - 6 apresenta os valores médios das variáveis após análise de pós-otimização, implementação dos PPL2' e PPL3'.

Comparando-se os valores das variáveis que definem as funções de utilidade obtidas para cada critério na análise pós-otimização, vê-se a grande diferença dos mesmos nas duas implementações. No critério $\mathrm{C} 1$, o valor extremo máximo na primeira implementação é de 0,143 e na segunda 0,010 . No critério $C 2$, o valor extremo máximo na primeira implementação é de 0,544 e na segunda 0,266 . No critério $\mathrm{C} 3$, o valor extremo máximo na primeira implementação é de 0,057 e na segunda 0,231 . No critério $\mathrm{C} 4$, o valor máximo extremo na primeira implementação é de 0,125 e na segunda 0,253 . No critério C5, o valor extremo máximo da variável na primeira implementação é de 0,049 e na segunda 0,012 . No critério C6, o valor extremo máximo na primeira implementação é de 0,082 e na segunda 0,228. 
A Tabela VI apresenta os valores limites das constantes de escala. A Coluna - 5 apresenta os limites superiores e a Coluna -6 apresenta os limites inferiores. As funções de utilidade dos seis critérios são apresentadas na Figura 1. Verifica-se a diferença das funções obtidas através da implementação das duas metodologias.
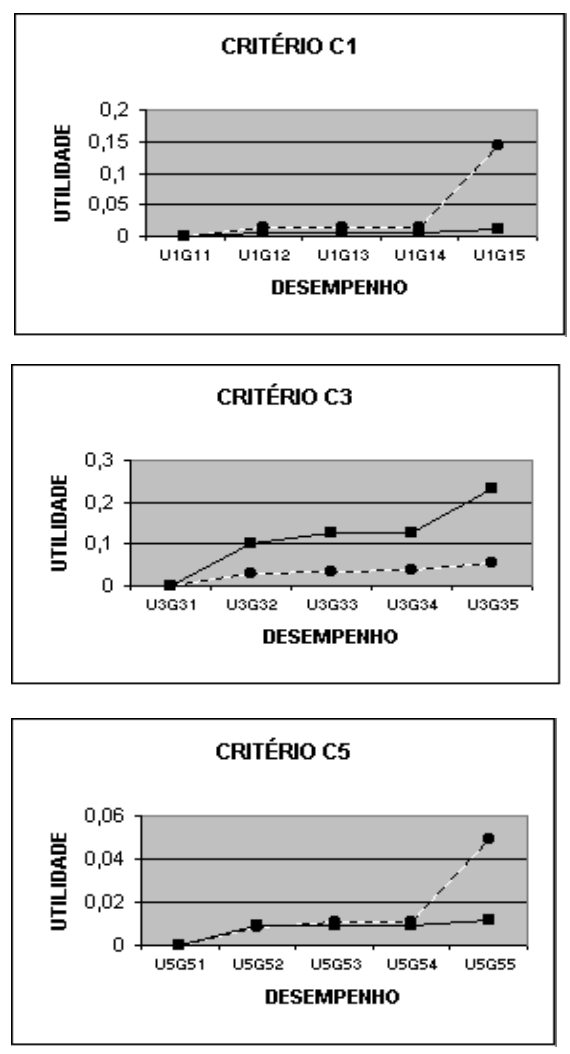

Figura 1 - Funções de Utilidade dos Critérios (Análise de Pós-Otimização), Sem Considerar (- - -) e Considerando (- ${ }^{-}$) as Constantes de Escala dos Critérios.

\section{Conclusões}

Empregaram-se dois métodos analíticos de apoio à decisão no estudo de caso relatado no presente artigo - o método UTA, na sua formulação original, que não leva em conta as constantes de escala atribuídos aos critérios pelos decisores, e, uma variante do referido método, que as considera para os critérios. As implementações dos modelos de programação linear foram feitas empregando o software Lindo.

Através dos resultados da análise pós-otimização de ambas as implementações, apresentadas Tabela V, verifica-se que a primeira implementação, empregando o método UTA original, atribui grande importância ao critério $\mathrm{C} 2$, e valoriza muito pouco os critérios C3 e C6. Já a segunda implementação, variante do método UTA, apresenta uma distribuição das constantes de escala mais realista, de acordo com as preferências dos decisores, determinando as 
constantes de escala de todos os critérios aproximadamente, com os mesmos valores que foram atribuídos pelos decisores. As funções de utilidade obtidas para cada critério nas duas implementações foram apresentadas na Figura 1.

A explicação para essas diferenças é que os modelos de programação linear, que descrevem o método UTA, apresentam soluções degeneradas, isto é, muitas soluções representam perfeitamente a ordenação fornecida pela CAD. Buscando restringir o conjunto de soluções dos modelos é que restrições para os valores extremos máximos para cada critério foram incorporadas aos mesmos.

Portanto, as funções de utilidades obtidas pela variante do método UTA, considerando as constantes de escala dos critérios, são mais coerentes com as preferências dos decisores, uma vez que, os mesmos são considerados pelo modelo através de duas restrições para cada critério. A inclusão de novas restrições ao modelo matemático, que descreve o método UTA, reduz o conjunto de soluções ótimas do problema, ao mesmo tempo em que busca soluções mais próximas das preferências dos decisores.

\section{Referências Bibliográficas}

(1) Barba-Romero, S.C. \& Pomerol, J.C. (1997). Decisiones Multicriterio - Fundamentos Teóricos y Utilización Práctica. Universidade de Alcalá, Espanha.

(2) CAD - Comissão de Avaliação de Docentes (1997). Regulamento da Comissão de Avaliação de Docentes da COPPE-UFRJ.

(3) CAD - Comissão de Avaliação de Docentes (1999). Apresentação dos Resultados da Avaliação CAD-98/99, COPPE-UFRJ.

(4) Gomes, L.F.A.M. (1998). Avaliação e Apoio à Tomada de Decisão. XVIII ENEGEP Encontro Nacional de Engenharia de Produção, Universidade Federal Fluminense, Niterói-RJ, Brasil.

(5) Keeney, R. \& Raiffa, H. (1993). Decisions with Multiple Objectives - Preference and Value Tradeoff. New York, Cambridge University Press.

(6) Jacquet-Lagrèze, E. \& Siskos, J. (1982). Assessing a set of Additive Utility Functions for Multicriteria Decision-Making, The UTA Method. European Journal of Operational Research, 10, 151-164.

(7) Rangel, L.A.D.; Gonzalez-Araya, M.C.; Lins, M.P.E. \& Gomes, L.F.A.M. (2000). Estimativa da Estrutura de Preferência da CAD-UFRJ para os Critérios de Avaliação de Docentes. XXXII SBPO - Sociedade Brasileira de Pesquisa Operacional, Viçosa-MG, Brasil.

(8) Roy, B. \& Bouyssou, D. (1993). Aide Multicritère à la Décision: Méthodes et Cas. Paris, Ed. Econômica.

(9) Vincke, Ph. (1989). L’Aide Muliticritère à la Décision. Bruxells, Éditions de l'Úniversité de Bruxells.

(10) Zopounidis, C. \& Dimitris, A.I. (1998). Multicriteria Decision Aid Methods for the Predictions of Business Failure. Kluwer Academic Publishers, Amsterdam. 


\section{Apêndice A: Uma representação do método UTA}

O método UTA é um método analítico do Apoio Multicritério à Decisão, desenvolvido por Jacquet-Lagrèze e Siskos em 1982 (Barba-Romero \& Pomerol, 1997). Esse método estima as funções de utilidade dos critérios, considerados no problema. As funções são agregadas em um único critério, chamado de critério de síntese. Para estimar as funções de utilidade aditiva é necessário uma ordenação de um conjunto de referência, subconjunto ou conjunto de alternativas, fornecida pelo decisor; e dos desempenhos das alternativas em relação aos critérios, isto é, a matriz de avaliação. Desta maneira, o método UTA começa por pedir ao decisor que ordene um conjunto de referência, empregando seu julgamento, isto é, sua preferência em relação aos elementos deste conjunto (Zopounidis \& Dimitris, 1998). Posteriormente, através da resolução de problema de programação linear determinam-se as variáveis, e desta maneira, as formas das funções de utilidade de cada critério. A função objetivo do modelo visa minimizar uma função dos erros associados às restrições que refletem às preferências, a priori, dado pelo decisor. A construção das funções de utilidade será tão mais fiel quanto maior for o número de alternativas que o agente de decisão consiga ordenar de forma subjetiva.

Para aplicar o método UTA assumem-se as bases axiomáticas, que estão por trás da teoria de utilidade multiatributo, buscando determinar as funções de utilidade aditiva (Keeney \& Raiffa, 1993). A seguir descreve-se resumidamente a teoria que aborda as funções de utilidade aditivas (Vincke, 1989), relacionando-as com o método UTA.

Considere-se um conjunto de alternativas "A", que é avaliado por uma família de critérios $\mathrm{g}=\left\{\mathrm{g}_{1}, \mathrm{~g}_{2}, \mathrm{~g}_{3}, \ldots, \mathrm{g}_{\mathrm{n}}\right\}$, sendo " $\mathrm{n}$ " o número de critérios e " $\mathrm{g}_{\mathrm{i}}$ " o desempenho no critério i. A agregação de todos os critérios em um único critério conduz à função de utilidade multiatributo, a qual representa-se por $\mathrm{U}(\mathrm{g})=\mathrm{U}\left(\mathrm{g}_{1}, \mathrm{~g}_{2}, \mathrm{~g}_{3}, \ldots, \mathrm{g}_{\mathrm{n}}\right)$.

Designa-se de "P" a relação de preferência estrita, e por I a relação de indiferença (Roy \& Bouyssou, 1985) entre duas alternativas, respectivamente.

Se $g(a)=\left[g_{1}(a), g_{2}(a), g_{3}(a), \ldots, g_{n}(a)\right]$ é a avaliação multicritério de uma alternativa a (Gomes, 1998), então valem as seguintes propriedades da função de utilidade U:

$$
\begin{aligned}
& \mathrm{U}[\mathrm{g}(\mathrm{a})]>\mathrm{U}[\mathrm{g}(\mathrm{b})] \Leftrightarrow \mathrm{aPb}, \\
& \mathrm{U}[\mathrm{g}(\mathrm{a})]=\mathrm{U}[\mathrm{g}(\mathrm{b})] \Leftrightarrow \mathrm{aI} b
\end{aligned}
$$

e a relação $\mathrm{R}=\mathrm{P} \cup \mathrm{I}$ define uma ordenação fraca das alternativas.

A função de utilidade é aditiva se é da forma abaixo:

$$
\mathrm{U}[\mathrm{g}]=\sum_{i=1}^{n} u_{i}\left(g_{i}\right)
$$

onde cada $\mathrm{u}_{\mathrm{i}}\left(\mathrm{g}_{\mathrm{i}}\right)$ é a utilidade marginal do desempenho $\mathrm{g}_{\mathrm{i}}$ no critério $\mathrm{i}$. Uma hipótese fundamental que é preciso não esquecer quando se aplica uma função de utilidade aditiva é a condição de independência mútua dos critérios em função das preferências (Keeney \& Raiffa, 1993).

Considere-se $\mathrm{g}_{\mathrm{i}}{ }^{*} \mathrm{o}$ valor mais alto e $\mathrm{g}_{\mathrm{i}^{*}} \mathrm{o}$ valor mais baixo em termos de preferência do critério i. Assumindo-se um não decréscimo das preferências em cada critério, então as utilidades marginais $u_{i}$ são funções monótonas crescentes. Portanto, pode-se normalizar as funções de utilidade dentro do intervalo $[0,1]$, tendo-se: 


$$
\begin{aligned}
& \sum_{i=1}^{n} u_{i}\left(g_{i}^{*}\right)=1, \\
& \mathrm{u}_{\mathrm{i}}\left(\mathrm{g}_{\mathrm{i}^{*}}\right)=0 \text { para todo } \mathrm{i} .
\end{aligned}
$$

O método UTA emprega a programação linear para determinar os valores das variáveis que descrevem as funções de utilidade de cada critério. Para fazer a implementação do método UTA são necessárias duas informações fundamentais em relação às alternativas: a avaliação multicritério e a ordenação fraca $\mathrm{R}$ definida em A', onde A' é subconjunto das alternativas presentes em A. Este conjunto A’ serve como conjunto de referência para que se interrogue o agente de decisão. Para cada par $(a, b) \in A^{\prime}$, o agente de decisão expressa sua preferência ou sua indiferença global.

Para cada alternativa de A', a função de utilidade calculada U' $[g(a)]$ difere da verdadeira $\mathrm{U}[\mathrm{g}(\mathrm{a})]$ por um erro $\sigma(\mathrm{a})$ :

$$
\mathrm{U}^{\prime}[\mathrm{g}(\mathrm{a})]=\sum_{i=1}^{n} u_{i}\left[g_{i}(a)\right]+\sigma(a), \quad \text { para todo } \mathrm{a} \in \mathrm{A}^{\prime}
$$

Considerando-se as relações de preferência (4) e de indiferença (5), bem como a função de utilidade (9), tem-se:

$$
\begin{aligned}
& \mathrm{U}^{\prime}[\mathrm{g}(\mathrm{a})]-\mathrm{U}^{\prime}[\mathrm{g}(\mathrm{b})] \geq \delta \Leftrightarrow \text { se o agente de decisão indica a P b } \\
& \mathrm{U}^{\prime}[\mathrm{g}(\mathrm{a})]-\mathrm{U}^{\prime}[\mathrm{g}(\mathrm{b})]=0 \Leftrightarrow \text { se o agente de decisão indica a I b }
\end{aligned}
$$

sendo $\delta>0$, um número real pequeno empregado a fim de separar significativamente duas classes da ordenação fraca R. Jacquet-Lagrèze e Siskos estabeleceram que este número tem que pertencer ao intervalo [1/10Q, 1/Q], sendo $\mathrm{Q}$ o número de classes de indiferença. Assumindo a existência de transitividade, o agente decisor só precisará fazer $(\mathrm{m}-1)$ comparações entre alternativas, onde m corresponde ao número de alternativas de A'.

Substituindo (9) em (10) e (11), tem-se:

$$
\begin{array}{ll}
\sum_{i=1}^{n}\left\{u_{i}\left[g_{i}(a)\right]-u_{i}\left[g_{i}(b)\right]\right\}+\sigma(a)-\sigma(b) \geq \delta & \Leftrightarrow \mathrm{aPb}, \\
\sum_{i=1}^{n}\left\{u_{i}\left[g_{i}(a)\right]-u_{i}\left[g_{i}(b)\right]\right\}+\sigma(a)-\sigma(b)=0 & \Leftrightarrow \mathrm{aIb},
\end{array}
$$

Considera-se as funções $u_{i}$ como sendo lineares por intervalos. Para definir tais intervalos, escolhem-se $\alpha_{i}$ pontos do intervalo $\left[g_{i^{*}}, g_{i}{ }^{*}\right.$ ] onde a função $u_{i}$ está definida. Cada ponto $g_{i}^{j}$ é determinado pela seguinte fórmula:

$$
g_{i}^{j}=g_{i^{*}}+\frac{j-1}{\alpha_{i}-1}\left(g_{i}^{*}-g_{i^{*}}\right)
$$

e j variando de 1 até $\alpha_{i}$, sendo o parâmetro $\alpha_{i}$ estabelecido pelo agente de decisão. Obtidos os pontos $g_{i}^{j}$, deve-se estimar os valores das variáveis $u_{i}\left(g_{i}^{j}\right)$. A utilidade marginal de uma alternativa a é aproximada por uma interpolação linear. Então, para $g_{i}(a) \in\left[g_{i}{ }^{j}, g_{i}{ }^{j+1}\right]$, tem-se:

$$
u_{i}\left[g_{i}(a)\right]=u_{i}\left(g_{i}^{j}\right)+\frac{g_{i}(a)-g_{i}^{j}}{g_{i}^{j+1}-g_{i}^{j}}\left[u_{i}\left(g_{i}^{j+1}\right)-u_{i}\left(g_{i}^{j}\right)\right]
$$


Satisfaz-se a hipótese da monotonicidade das preferências das utilidades marginais $\mathrm{u}_{\mathrm{i}}\left(\mathrm{g}_{\mathrm{i}}\right)$, exigida pelo método, através do seguinte conjunto de restrições:

$$
u_{i}\left(g_{i}^{j+1}\right)-u_{i}\left(g_{i}^{j}\right) \geq s_{i}, \quad \mathrm{j}=1,2,3, \ldots, \alpha_{i}-1, \quad \mathrm{i}=1,2,3, \ldots, \mathrm{n} .
$$

onde $\mathrm{s}_{\mathrm{i}}>0$ é o limite de indiferença definido em cada critério i.

Estima-se as variáveis que representam as utilidades $\mathrm{u}_{\mathrm{i}}\left(\mathrm{g}_{\mathrm{i}}^{\mathrm{j}}\right)$ e os erros $\sigma(\mathrm{a})$ por meio de programação linear, considerando as restrições apresentadas anteriormente, quais sejam: restrições segundo as preferências e as indiferenças entre as alternativas a $\in A^{\prime}$, restrições que garantem a hipótese da monotonicidade das utilidades marginais, restrições aos limites superiores e inferiores de cada critério; e restrições relativas a hipótese de não negatividade das variáveis. A função objetivo do problema de programação linear (PPL) corresponde à minimização dos erros $\sigma(a)$ associados às utilidades de cada alternativa $a \in A$ '. Assim, a formulação matemática do PPL é:

(PPL1) Min $\quad F=\sum_{a \in A^{\prime}} \sigma(a)$

Sujeito a:

$$
\begin{array}{ll}
\sum_{i=1}^{n}\left\{u_{i}\left[g_{i}(a)\right]-u_{i}\left[g_{i}(b)\right]\right\}+\sigma(a)-\sigma(b) \geq \delta \Leftrightarrow & \text { se a P* b, } \\
\sum_{i=1}^{n}\left\{u_{i}\left[g_{i}(a)\right]-u_{i}\left[g_{i}(b)\right]\right\}+\sigma(a)-\sigma(b)=0 \Leftrightarrow & \text { se a I* } \mathrm{b}, \\
u_{i}\left(g_{i}^{j+1}\right)-u_{i}\left(g_{i}^{j}\right) \geq s_{i}, & \text { para todo i e j, } \\
\sum_{i=1}^{n} u_{i}\left(g_{i}^{*}\right)=1 & \text { para todo i } \\
\mathrm{u}_{\mathrm{i}}\left(\mathrm{g}_{\mathrm{i}^{*}}\right)=0, & \text { para todo i e j } \\
\mathrm{u}_{\mathrm{i}}\left(\mathrm{g}_{\mathrm{i}}^{\mathrm{j}}\right) \geq 0, & \text { para todo a } \in \mathrm{A}^{\prime} .
\end{array}
$$

A solução ótima de PPL1 será aquela(s) que obtenha(m) o melhor valor para a função objetivo. Seja $\mathrm{F}^{*}$ o valor ótimo. A experiência de uso do método UTA indica que, muitas vezes, podem existir soluções aceitáveis $\left(\mathrm{F}>\mathrm{F}^{*}\right)$ que apresentam, por exemplo, um melhor desempenho em relação às distâncias de Kendall ou Spearman que a solução ótima. Isto é, a ordem fraca R' obtida por soluções aceitáveis está mais próxima da ordem fraca $R$ determinada pelo agente de decisão que a ordem fraca determinada pela solução ótima. Por este motivo, o método UTA não para com a obtenção de uma solução ótima, senão que explora, através de eficientes procedimentos de pós-otimização, os vértices de um novo poliedro, o qual obtém-se acrescentando a restrição seguinte ao conjunto de restrições de PPL1:

$$
\mathrm{F} \leq \mathrm{F}^{*}+\mathrm{k}\left(\mathrm{F}^{*}\right)
$$

A restrição (25) estabelece que a função objetivo $F$ não deve ultrapassar $F^{*}$ além de um limite positivo $\mathrm{k}\left(\mathrm{F}^{*}\right)$, sendo $\mathrm{k}\left(\mathrm{F}^{*}\right)$ uma proporção muito pequena de $\mathrm{F}^{*}$. 
Os vértices deste novo poliedro correspondem às funções de utilidade onde um ou mais critérios atingem um valor extremo (ou máximo ou mínimo). Assim, a exploração do novo poliedro pode ser feita resolvendo os seguintes problemas de programação linear:

$$
\text { (PPL2) Min } u_{i}\left(g_{i}^{*}\right) \quad \text { e } \quad(P P L 3) M a x u_{i}\left(g_{i}^{*}\right), \quad i=1, \ldots, n .
$$

Os PPL2 e PPL3 estão sujeitos às restrições (18), (19), (20), (21), (22), (23), (24) e (25). Finalmente, o método UTA propõe-se calcular as médias das funções de utilidade marginais $\mathrm{u}_{\mathrm{i}}\left(\mathrm{g}_{\mathrm{i}}{ }^{\mathrm{j}}\right)$ obtidas pela análise de pós-otimização. Com estas médias pode-se avaliar a $\mathrm{U}(\mathrm{g}(\mathrm{a}))$ de todas as alternativas $a \in A$, sejam ou não estas alternativas do conjunto de referência A'. 